\title{
Ruido electroquímico de la erosión-corrosión en cobre: su relación con los parámetros hidrodinámicos ${ }^{(\bullet)}$
}

\author{
I. Castañeda*, M. Romero**, J. M. Malo*** y J. Uruchurtu*, **
}

\begin{abstract}
Resumen
En este trabajo se presentan los resultados de los efectos del deterioro a la integridad superficial, de muestras de cobre de pureza comercial, debido al fenómeno de erosión-corrosión en presencia de un fluido que contiene partículas de sílice de diversos tamaños, usando la técnica de ruido electroquímico. Esto, en función de los parámetros hidrodinámicos del sistema (movimiento del fluido). Utilizando un electrodo de cilindro rotatorio (CR) modificado con tres anillos, se posibilitó a manejarlo empleando dos velocidades de giro ( 880 y 1.750 rpm y números de Reynolds de $1.486 \operatorname{Re~y~} 2.972 \mathrm{Re}$, respectivamente). Se encontró patrones característicos de ruido electroquímico en función del régimen de flujo laminar y de transición turbulenta; así como de intensidades de daño en la superficie del material, teniendo una relación con los patrones de ruido electroquímico observados. Se presenta un ataque más uniforme y un aumento del impacto para las partículas de mayor tamaño y una intensidad menor de erosión en la superficie expuesta, con una forma de ataque más localizado para las partículas de menor tamaño. El estudio del fenómeno de erosión-corrosión presenta patrones de identificación de ruido electroquímico de corriente y de potencial característicos, de acuerdo al régimen de flujo laminar o de transición turbulenta y a la adición de diferentes tamaños de partículas sólidas de alúmina y sílice.
\end{abstract}

\section{Electrochemical noise of the erosion-corrosion of copper in relation with its hydrodynamic parameters}

\begin{abstract}
This work presents the electrochemical noise results obtained of the surface degradation on copper, due to erosion corrosion phenomena, which were a function of the hydrodynamic parameters of the system (fluid movement). A modified rotating cylinder (RC) comprising three ring electrodes under two rotating speeds ( 880 and $1750 \mathrm{rpm}$ with a Reynolds numbers $1486 \mathrm{Re}$ and $2972 \mathrm{Re}$, respectively) were used. Characteristic electrochemical noise spectra as a function of the hydrodynamic parameters were found, as well as surface attack intensities the noise signal. An increase and a more uniform attack due to particle impact was related to larger particle size and lesser erosion corrosion intensity, in the form of more localized attack over the surface, was obtained for smaller ones. Erosion corrosion attack presents characteristic electrochemical current and potential noise signals, according to the laminar or transitional turbulent regime and particle size added.
\end{abstract}

Keywords

Erosion corrosion; Rotating disk; Spectra; Electrochemical noise.

\section{INTRODUCCIÓN}

La erosión-corrosión es la aceleración e incremento en la tasa de deterioro o ataque en un sustrato metálico, debido al movimiento relativo entre un fluido corrosivo que contiene partículas erosivas y la superficie metálica. Mecánicamente, se puede asociar la eliminación física de películas protectoras seguida de la de material metálico de la superficie del sustrato, que dan como resultado un subsecuente incremento de la corrosión electroquímica.

Para que el deterioro por erosión-corrosión suceda en un material dado, a menudo se opina que es necesario exceder una velocidad crítica del fluido ${ }^{[1]}$. El daño mecánico por el impacto del fluido impone deformaciones significativas por concentración local de

\footnotetext{
(•) Trabajo recibido el día 12 de Enero de 2010 y aceptado en su forma final el día 14 de Abril de 2010.

* Centro de Investigaciones en Ingeniería y Ciencias Aplicadas, CIICAp., Av Universidad 1001, Colonia Chamilpa, Cuernavaca, Morelos, México.

** Universidad Autónoma Metropolitana, UAM Atzcapotzalco, Avenida del Rosario Atzcapotzalco, México D.F.

*** Instituto de Investigaciones Eléctricas, IIE Interior, Internado Palmira Colonia, Palmira, Temixco, Morelos, México.
} 
esfuerzos o variaciones de presión sobre la superficie de la película protectora y/o del material subyacente a la superficie. La cinética de la erosióncorrosión puede aumentar debido a la presencia de partículas (sólidas o burbujas de gas) que son impactadas, como resultado de condiciones hidrodinámicas diversas, asociadas a flujos multifásicos. La topografía de las superficies afectadas por erosióncorrosión puede ser circular (hoyos, huecos) poco profundos u orificios en forma de herraduras o alguna otra forma de ataque local relacionado con la dirección del flujo ${ }^{[2]}$.

En la pasada década se desarrolló una búsqueda más detallada de medios de detección y seguimiento del grado de daño debido a la corrosión, en diferentes metales para, así, poder proponer mecanismos y optimizar procesos o el uso de medidas de protección que disminuyan el deterioro de los materiales, a fin de reducir accidentes y costes de producción.

El ruido electroquímico es una técnica sensible en la detección de corrosión localizada y útil bajo condiciones de efectos electroquímico-mecánicos. Existen diversos métodos de análisis de las señales de ruido electroquímico, tales como: visual, estadístico, análisis en frecuencia y los métodos dinámicos no lineales ${ }^{[3-7]}$. La investigación ha mostrado las bondades inherentes a la técnica durante la monitorización, en línea y tiempo real, de la corrosión en procesos industriales ${ }^{[8-10]}$. El principio de medición empleado en el presente trabajo aplica la técnica de ruido electroquímico, que se utiliza desde hace 20 años en forma creciente, en el seguimiento y control de procesos de corrosión y cuya principal característica es ser una técnica "no destructiva" [11 y 12].

No existen muchas aplicaciones de la técnica de ruido electroquímico al estudio de los fenómenos que involucran corrosión erosión: por ejemplo, Puget ${ }^{[11}$ y 12] , quién estudió los mecanismos de degradación de revestimientos, sometidos a partículas de arena a una velocidad de $4 \mathrm{~m} / \mathrm{s}$, en el cual no se encontró sinergismo (dependencia) entre la erosión y corrosión. También, Wood ${ }^{[13}$ y 14], que encontró oscilaciones en las señales de ruido electroquímico en potencial, relacionando un mayor efecto del fenómeno de erosión con el proporcionado por la corrosión. Teniendo así la aportación de la técnica de ruido electroquímico.

Este trabajo presenta resultados del efecto erosivo de partículas sobre cobre, bajo diferentes regímenes de flujo laminar y de transición turbulenta, demostrando las ventajas del uso del ruido electroquímico para evaluar el fenómeno de erosión-corrosión y su relación con los parámetros estudiados.

\section{MATERIALES Y MÉTODOS}

\subsection{Preparación del electrodo de trabajo}

Como material para formar los electrodos de trabajo se utilizó cobre comercial (tubería de cobre tipo M, norma B88) en forma de anillos. Para conformar el diseño de los electrodos se manejaron tres anillos de cobre con las mismas características: de 0,9 cm de diámetro exterior, $0,7 \mathrm{~cm}$ de diámetro interior y 5 $\mathrm{cm}$ de ancho, cortados con un cortador de tubos de cobre, a los que se impartió un pretratamiento metalográfico estándar de desbaste, con lija de carburo de silicio del grado $200 \mathrm{al} 600$, a fin de eliminar las rebabas generadas en el corte y obtener una superficie limpia.

El arreglo tiene como propósito montar los tres anillos en el mismo porta-electrodo, tal que, uno trabajará como electrodo de trabajo 1 (WE1, por sus siglas en inglés); otro, como electrodo de trabajo 2 (WE2) y el tercero como electrodo de referencia (RE); la configuración de tres electrodos idénticos es comúnmente utilizada para la medición de ruido electroquímico.

Para la preparación del electrodo de trabajo utilizado para el cilindro rotatorio (CR), se mecanizó un porta-electrodo de Nylamid, para lo cual fue necesario respetar las dimensiones de los anillos cortados previamente, con el fin de que la cara exterior de los anillos quedara totalmente expuesta al ambiente de experimentación, con pequeños orificios que atravesaran al porta-electrodos. Por estos orificios pasan conductores metálicos (alambres de cobre) hacia la parte interna, logrando continuidad del contacto entre el anillo a través del alambre conductor hacia el instrumental del cilindro rotatorio. El electrodo del cilindro rotatorio con los anillos del material de trabajo de cobre, se sumergió en agua destilada conteniendo partículas sólidas de sílice y alúmina. El arreglo esquemático se presenta en la figura 1 donde, en el porta-electrodos con los anillos de cobre, se coloca una rondana de plástico y las uniones de los anillos con el porta electrodos se cubren con silicona para evitar que el electrólito penetre hacia la cara interna y se asegure el contacto de la solución, sólo, en la cara externa de los anillos.

\subsection{Arreglo de la celda electroquímica}

Debido a la manipulación previa de las probetas de trabajo, es preciso adaptar el porta-electrodo en la flecha del disco rotatorio, integrando, así, una sola pieza y evitando el desequilibrio entre la unión de la 


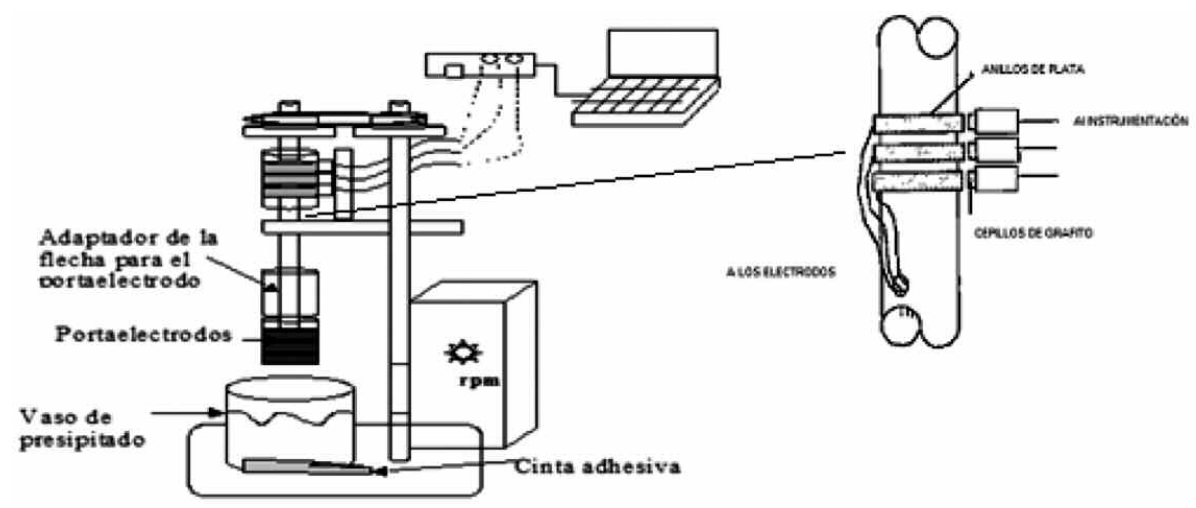

Figura 1. Arreglo experimental y electrodo de anillos.

Figure 1. Experimental setup and ring electrode.

flecha y-porta-electrodo. La unión se realiza conectando el conector macho del porta-electrodo y el conector hembra de la flecha; estas dos piezas también se adhieren por medio de silicona de uso comercial[11]. Como celda de reacción se trabajó en un vaso de precipitados de vidrio, de $600 \mathrm{ml}$, como se muestra en la figura 1.

\subsection{Preparación de soluciones}

Como electrólito se utilizó agua destilada a pH 6, procurando una cantidad de volumen constante en cada prueba de $300 \mathrm{ml}$. La solución se desaireó con $\mathrm{N}_{2}$, durante media hora antes de comenzar las pruebas para enfatizar los efectos mecánicos y del transporte de masa en el fenómeno. Para ello se utilizaron 2 tipos de partículas sólidas (sílice y alúmina) y, cada una, con tres diferentes tamaños. Para establecer un régimen de flujo laminar y uno de transición turbulenta, se utilizaron las velocidades de rotación de 800 y 1.760 rpm con 1.486 Re y 2972 Re, respectivamente ${ }^{[23]}$.

Para la medición de ruido electroquímico con los anillos de trabajo de cobre adaptados al disco rotatorio, se empleó como equipo de medición un amperímetro de resistencia cero (ZRA, por sus siglas en inglés) de ACM Instruments. Unos cuantos segundos después de haber iniciado la prueba con el ACM Instruments, se vierten $30 \mathrm{~g}$ de partículas sólidas dentro de la celda con agua destilada; se esperan $15 \mathrm{~min}$ para la estabilización del sistema (potencial libre y/o patrón de flujo) y se procede a adquirir los datos. Este procedimiento se repitió para cada tipo de partícula y para cada tamaño de ellas.

Para tener una referencia de comparación (blan$\mathrm{co})$, se realizaron estas mismas pruebas de experimentación, solo que se procedió a medir el ruido electroquímico en condiciones estáticas del electrólito, es decir, el sistema se conformó de agua destilada y anillos de cobre sin rotación del eje, con los electrodos. Además, se procedió a medir ruido electroquímico en condiciones de movimiento sin partículas sólidas, es decir sólo agua destilada y anillos de cobre en rotación.

\subsection{Partículas erosivas}

Como partículas erosivas se utilizaron partículas de diferentes tamaños: 0,3, 1 y 74-179 (malla 80-200) mm de alúmina $\left(\mathrm{Al}_{2} \mathrm{O}_{3}\right)$ con forma redondeada; $\mathrm{y}$ menor a 250 (malla 60), menores a 590 (malla 30) y mayores a $590 \mathrm{~mm}$ de sílice $\left(\mathrm{SiO}_{2}\right)$ con forma irregular (Fig. 2). Se hizo un tamizado para separar los tamaños de partículas con mallas de diferente tamaño de apertura.

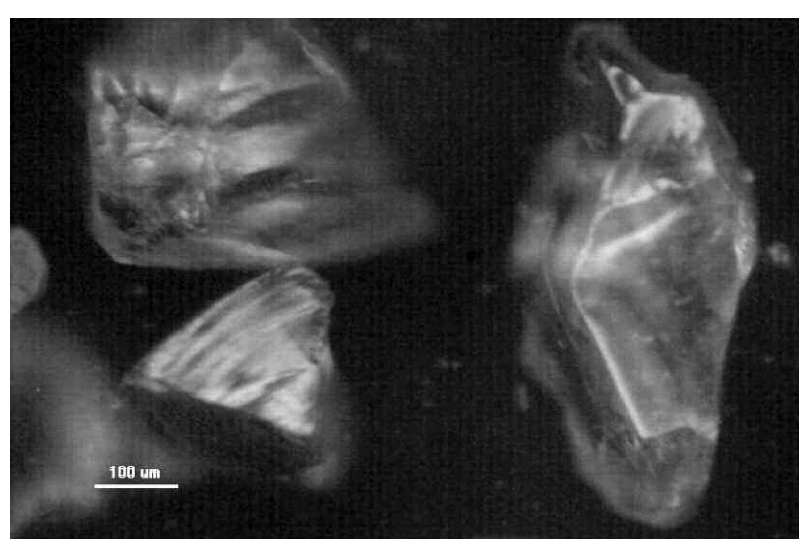

Figura 2. Partículas de sílice.

Figure 2. Silica particles. 
RUIDO ELECTROQUIIMICO DE LA EROSIÓN-CORROSIÓN EN COBRE: SU RELACIÓN CON LOS PARÁMETROS HIDRODINÁMICOS ELECTROCHEMICAL NOISE OF THE EROSION-CORROSION OF COPPER IN RELATION WITH ITS HYDRODYNAMIC PARAMETERS

\subsection{Mediciones}

Se realizaron pruebas de ruido electroquímico, en series de potencial y corriente, de 4096 datos, tomados durante el período de exposición, diferenciando las señales de ruido en tres estados del sistema: estático, dinámico y dinámico con partículas sólidas. Se obtuvo la resistencia de ruido a partir de las desviaciones estándar de potencial divididas entre las desviaciones estándar de corriente.

\section{RESULTADOS Y DISCUSIÓN}

Para establecer el patrón (blanco), se midió el ruido electroquímico en corriente y en potencial y se calculó la resistencia como se estableció más arriba. En las figuras 3, 4 y 5 se muestran los resultados, bajo tres diferentes condiciones de exposición. La primera condición, consiste en exponer los tres anillos de cobre colocados en el cilindro rotatorio (CR) en agua destilada, en condiciones estáticas para minimizar el

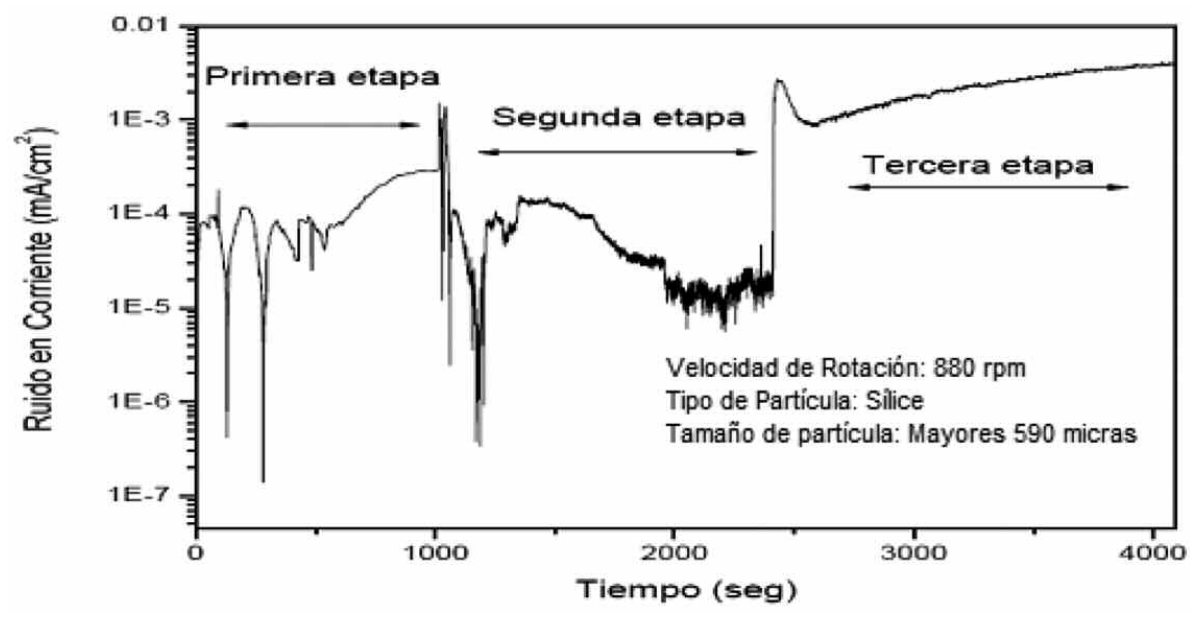

Figura 3. Ruido en corriente para condiciones de flujo laminar, con velocidad de giro de $880 \mathrm{rpm}$, y tamaños de partículas de sílice mayores de $590 \mu \mathrm{m}$.

Figure 3. Electrochemical current noise for the laminar flow conditions with 880 rpm rotating speed, and silicon particles greater than $590 \mu \mathrm{m}$.

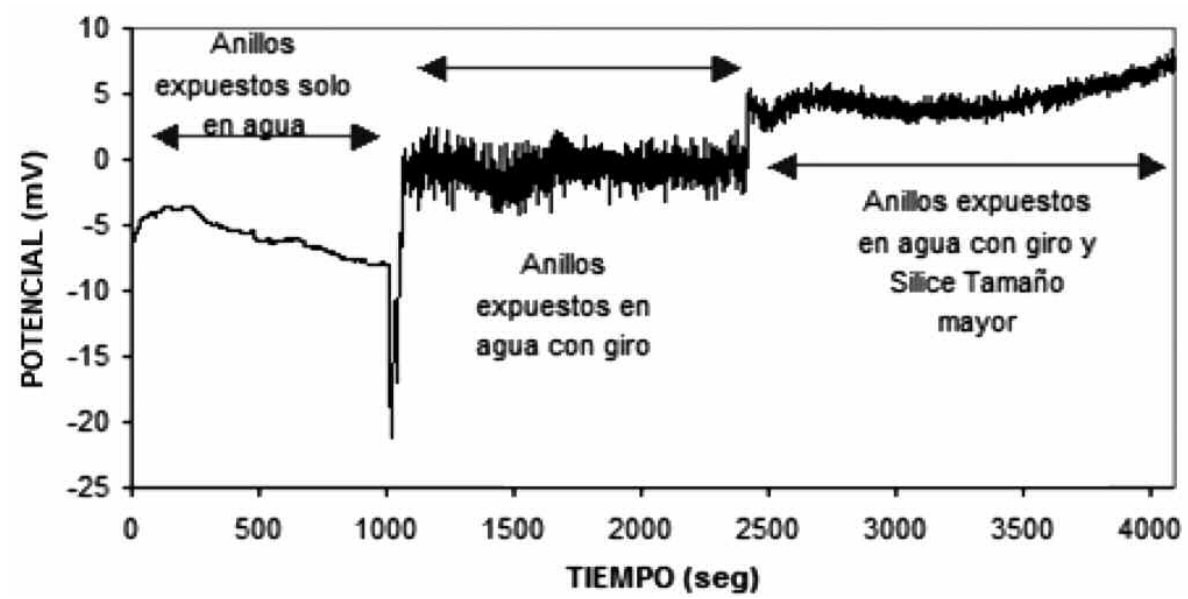

Figura. 4. Ruido en potencial para condiciones de flujo laminar, con velocidad de giro de $880 \mathrm{rpm}$, partículas de sílice y con tamaños mayores de $590 \mu \mathrm{m}$.

Figure 4. Electrochemical potential noise for the laminar flow conditions with $880 \mathrm{rpm}$ rotating speed, and silicon particles greater than $590 \mu \mathrm{m}$. 


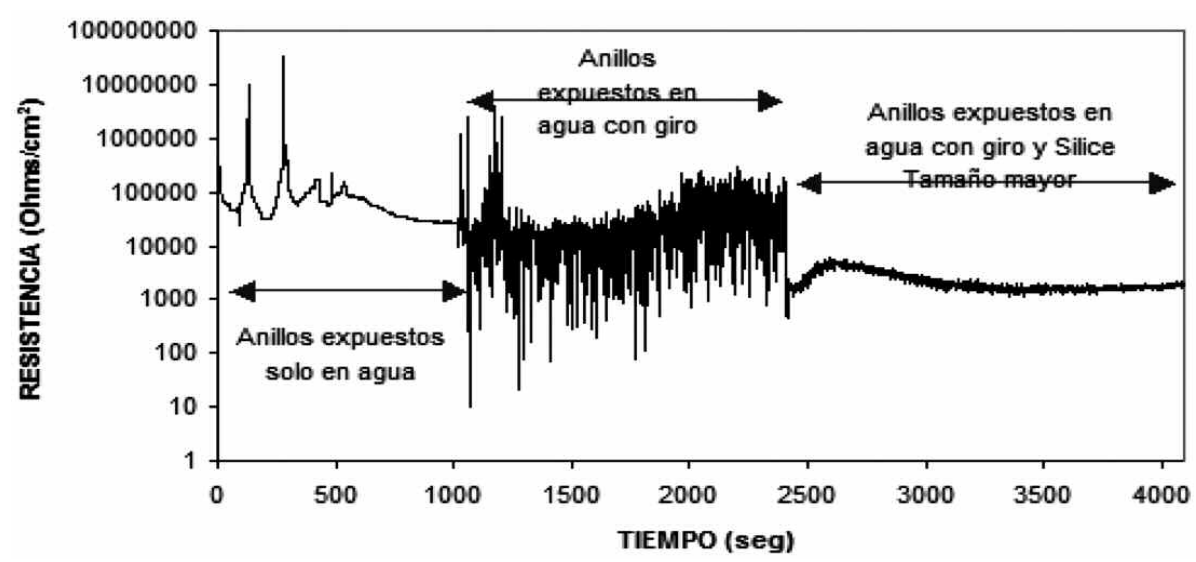

Figura 5. Resistencia al ruido para condiciones de flujo laminar, con velocidad de giro de $880 \mathrm{rpm}$, partículas de sílice y con tamaños mayores de $590 \mu \mathrm{m}$.

\section{Figure 5. Electrochemical noise resistance for the laminar flow conditions with $880 \mathrm{rpm}$ rotating speed, and silicon particles greater than $590 \mu \mathrm{m}$.}

efecto agresivo de la solución. Sin embargo, se presentan algunos momentos transitorios, asociados, quizás, a rompimientos por tensiones mecánicas, de la capa pasiva (efectos localizados) del cobre en este medio,. En esta condición, el ruido en corriente presenta oscilaciones, observándose una tendencia a la estabilización, cercana a $1 \times 10^{-4} \mathrm{~mA} \mathrm{~cm}^{-2}$. A partir de los $1.000 \mathrm{~s}$, se encendió el CR (880 rpm); los resultados mostraron una serie de valores transitorios que volvían al estado estacionario con una disminución en el valor de corriente cercano a $1 \times 10^{-5} \mathrm{~mA} \mathrm{~cm}^{-2}$. Se presentaron algunas pequeñas oscilaciones sobrepuestas, de alta frecuencia pero de muy baja amplitud, ausentes en condiciones estáticas, debido al efecto de la difusión por la agitación de la solución. A los $2.400 \mathrm{~s}$ se agregaron las partículas de sílice: se observó una oscilación, con un incremento sostenido del nivel de corriente mayor a $1 \mathrm{x}^{-3} 0^{-3} \mathrm{~mA} \mathrm{~cm}^{-2}$, debido a los efectos mecánicos sobre la superficie, por las partículas. Es clara la diferencia para las tres condiciones de experimentación.

Esto, es igualmente notable para el ruido en potencial que se presenta en la figura 4, donde se observa un comportamiento inverso al del ruido en corriente y se distinguen, claramente, las tres condiciones. En condiciones estáticas, no se presentaron oscilaciones de alta frecuencia en el régimen estático sino un comportamiento más sinuoso en baja frecuencia, asociado a procesos de difusión. Al encender el CR, el valor de potencial en estado estacionario cambió de $-8 \mathrm{mV}$ y, después de un trransitorio, alcanzó los $0 \mathrm{mV}$. Aparecieron oscilaciones de alta frecuencia y poca amplitud sobrepuestas y, al agregar las partículas, se produjo un incremento subsecuente de $5 \mathrm{mV}$, con tendencia a crecer, del valor en estado estacionario, mostrando ruido sobrepuesto de menor amplitud respecto al régimen dinámico sin partículas.

La resistencia de ruido electroquímico calculada en función del tiempo se presenta en la figura 5, observándose un comportamiento muy claro para los tres estados. En condiciones estáticas, se presentó una serie de mediciones de alta amplitud con un incremento de 2 órdenes de magnitud y baja frecuencia que regresaron al valor estacionario. Al encender el $\mathrm{CR}$, aparecieron valores transitorios en ambos sentidos, de alta amplitud y frecuencia, que disminuyeron en intensidad (amplitud) al agregar las partículas de sílice. A lo largo de la prueba y a medida que cambiaron las condiciones, el valor promedio (estado estacionario) de resistencia de ruido disminuyó de $1 \times 10^{5}$

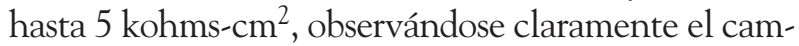
bio de valor en función de las condiciones de impacto de las partículas (mayor erosión-corrosión).

Se realizó un análisis espectral mediante la transformada rápida de Fourier (FFT), para las tres condiciones de experimentación, por separado, de las series de ruido en potencial y corriente en función del tiempo, que se presentan en la figura 6. A partir de la densidad de potencia espectral (Power Spectral Density, como se conoce en inglés) se suele obtener información diversa relacionada con el proceso de corrosión que generó la señal de ruido estudiada, así como el espectro de la impedancia de ruido electroquímico ${ }^{[12-14]}$. Para determinar los espectros y eliminar el efecto de corrimiento de corriente directa (DC), se eliminó la tendencia de las series de ruido a partir de un ajuste por mínimos cuadrados, ya descrito en la literatura ${ }^{[9-13]}$. 
RUIDO ELECTROQUÍMICO DE LA EROSIÓN-CORROSIÓN EN COBRE: SU RELACIÓN CON LOS PARÁMETROS HIDRODINÁMICOS ELECTROCHEMICAL NOISE OF THE EROSION-CORROSION OF COPPER IN RELATION WITH ITS HYDRODYNAMIC PARAMETERS
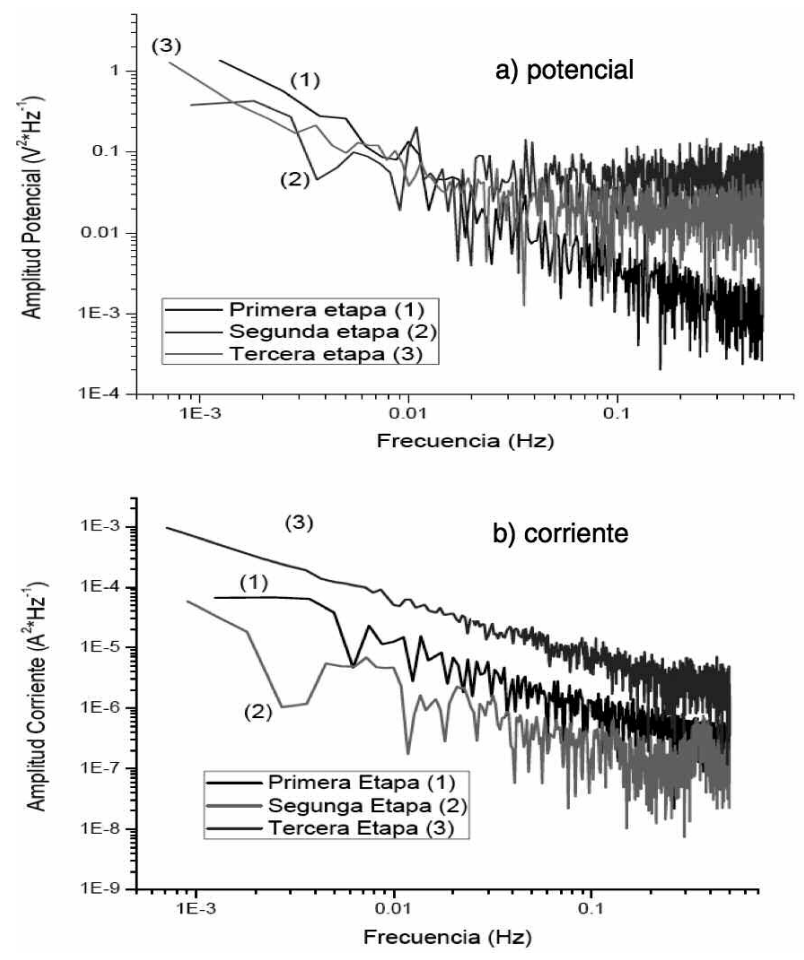

Figura 6. Espectro en a) potencial y b) corriente para condiciones de flujo laminar.

\section{Figure 6. Potential and current noise spectra for laminar flow conditions.}

El espectro de ruido en potencial presenta la mayor amplitud en el límite de baja frecuencia, para la primera etapa y amplitudes similares para la segunda y tercera etapas. Las amplitudes para altas frecuencias se ven incrementadas en función del régimen de flujo laminar y de transición turbulenta $(\mathrm{Re}=1.486$ y 2.972, respectivamente) y de la presencia de las partículas. El espectro en corriente presenta la condición de mayor amplitud de ruido en el límite de baja frecuencia para la tercera etapa con un nivel mayor, mientras que la menor amplitud se presenta para la segunda etapa. Sin embargo, en alta frecuencia los valores de amplitud se alinean de menor a mayor de acuerdo con las etapas estudiadas. Esto sugiere una relación del nivel de ruido en función de las condiciones dinámicas y ataque superficial.

Esto, se observa con mayor claridad en la figura 7, que resulta de dividir el espectro de potencial entre el de corriente, con lo que se obtiene el espectro de la impedancia de ruido en baja frecuencia: se puede observar un incremento en la impedancia con relación a las condiciones hidrodinámicas. Los tres espectros presentaron una pendiente suave en bajas frecuencias, que después se torna un valor constante en altas frecuencias y que se relaciona con el rompimiento de la capa pasi$\mathrm{va}^{[4]}$. Sin embargo el espectro correspondiente al de la

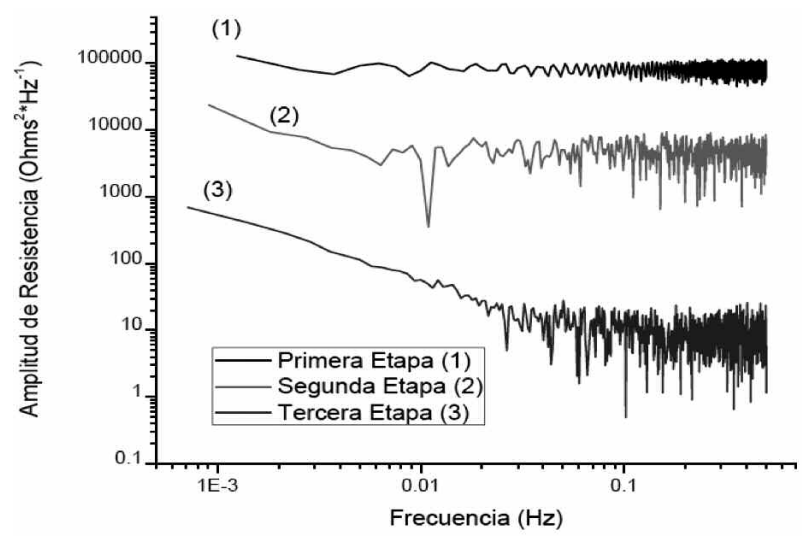

Figura 7. Impedancia de ruido electroquímico para condiciones de flujo laminar

Figure 7. Electrochemical noise impedance for laminar flow conditions.

rotación con partículas presenta mayores variaciones en sus valores para estas frecuencias, las que son asociadas a los impactos sobre la superficie del electrodo ${ }^{[14]}$. El mayor efecto de las diferentes condiciones se registra en los valores promedio de los parámetros de ruido electroquímico, los cuales varían en función de las condiciones experimentales como los efectos de transportes y los mecánicos ${ }^{[9-11]}$.

La constante de tiempo $(\tau)$ para estas tres condiciones se puede calcular de acuerdo a lo siguiente:

$$
\begin{aligned}
& 2 \pi f_{c}=1 / \tau \\
& \tau=R_{n} C_{d l}
\end{aligned}
$$

dónde, $\mathrm{f}_{\mathrm{c}}$ es la frecuencia de corte del espectro, $\mathrm{R}_{\mathrm{n}}$ es la resistencia de ruido, $\mathrm{C}_{\mathrm{dl}}$ es la capacitancia de la doble capa (o pseudo-capacitancia) y $\tau$ es la constante de tiempo del proceso involucrado. A medida que se modifican las condiciones, la frecuencia de corte y la capacitancia aumentan, la resistencia disminuye y la constante de tiempo se hace menor. Esto es debido al aumento en la frecuencia de impacto de las partículas por la mayor velocidad de rotación, y a la exposición de material fresco (rompimiento de la capa pasiva) a la solución, consecuentemente se modifican los valores de los parámetros electroquímicos. Los valores de la constante de tiempo y de los parámetros electroquímicos están relacionados con las condiciones de la superficie metálica. Se observa el efecto que tiene la agitación en la difusión del oxígeno y el control del proceso en presencia de las partículas.

En esta etapa del trabajo, se realizaron pruebas, para observar el comportamiento del fenómeno de erosión-corrosión con la variable velocidad de 
Tabla I. Parámetros electroquímicos y constante de tiempo para condiciones de flujo laminar, a partir de las ecuaciones 1 y 2

Table I. Electrochemical parameters and time constant for laminar flow conditions, from equations 1 and 2

\begin{tabular}{lcccc}
\hline Condiciones & $\mathbf{f}_{\mathbf{c}}(\mathbf{m H z})$ & $\mathbf{R}_{\mathbf{n}}\left(\mathbf{k} \Omega \mathbf{c m}^{2}\right)$ & $\mathbf{C}_{\mathrm{dl}}\left(\mu \mathbf{F c m} \mathbf{-}^{2}\right)$ & $\tau \mathbf{m s e g}$ \\
\hline Estático & 3 & 200 & 265 & 53,07 \\
Con giro & 5 & 30 & 1.061 & 31,84 \\
Con partículas & 25 & 0,950 & 6.694 & 6,36 \\
\hline
\end{tabular}

rotación ${ }^{[15]}$. En estas pruebas, se utilizaron las condiciones de prueba realizadas en la etapa anterior sólo que, para este caso, se modificó la velocidad de giro en el disco rotatorio, al doble de la velocidad anterior $(1.760 \mathrm{rpm})$ para, así, tener un régimen de flujo de transición turbulenta ${ }^{[23]}$. Se manejaron 3 tamaños de partículas de sílice, como se indicó en la sección respectiva.

Las figuras 8 y 9 , presentan el ruido electroquímico en corriente y potencial (tendencia removida)
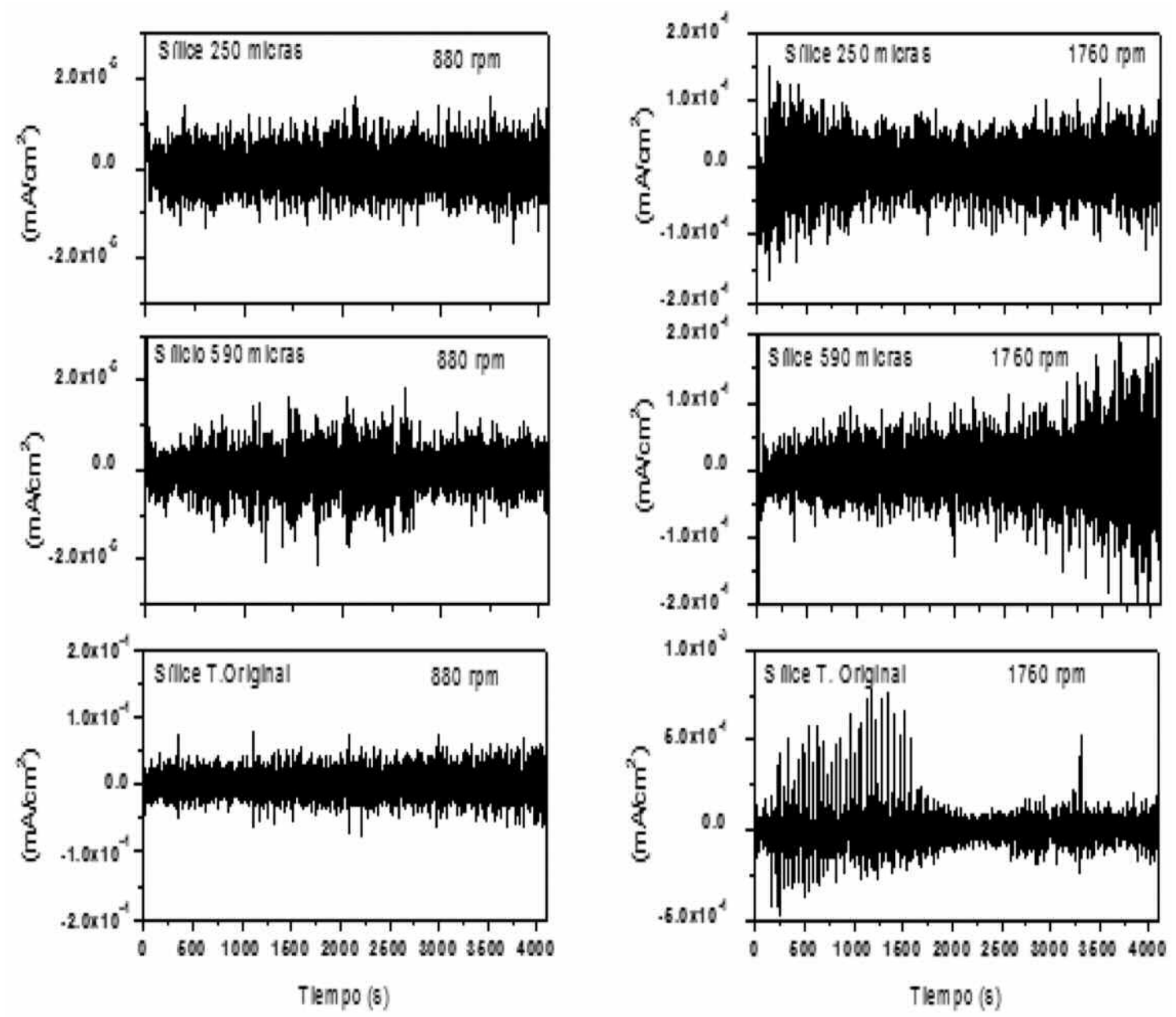

Figura 8. Ruido en corriente para diferentes tamaños de partícula y velocidades de rotación.

Figure 8. Current noise for different particle size and rotation speeds. 
RUIDO ELECTROQUÍMICO DE LA EROSIÓN-CORROSIÓN EN COBRE: SU RELACIÓN CON LOS PARÁMETROS HIDRODINÁMICOS ELECTROCHEMICAL NOISE OF THE EROSION-CORROSION OF COPPER IN RELATION WITH ITS HYDRODYNAMIC PARAMETERS
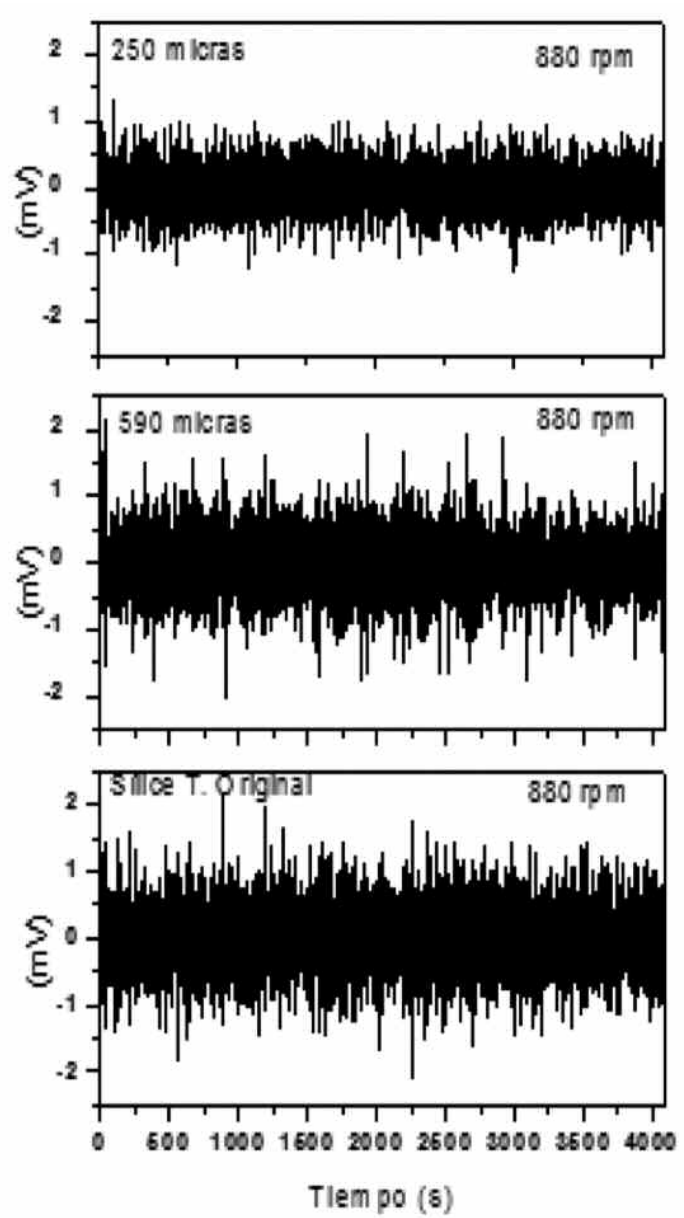
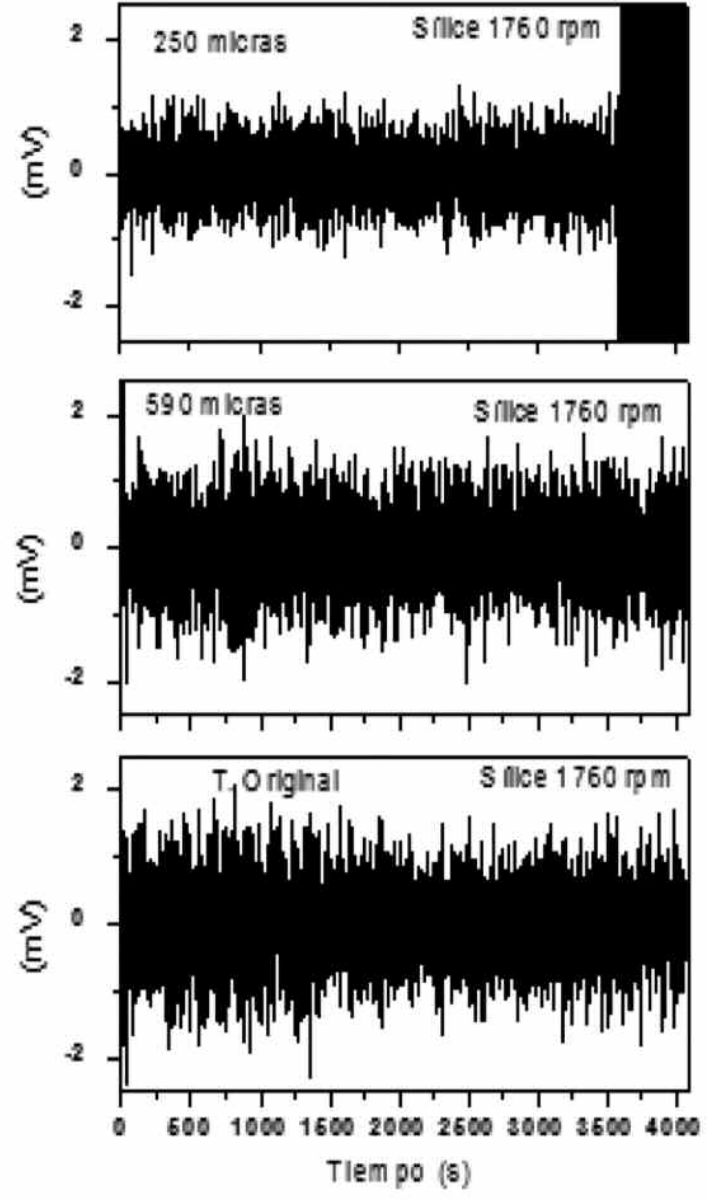

Figura 9. Ruido en potencial para diferentes tamaños de partícula y velocidades de rotación.

Figure 9. Potential noise for different particle size and rotation speed.

obtenido para las dos diferentes velocidades y tamaño de partícula. Se puede observar los cambios en los valores de amplitud de las fluctuaciones observadas, así como el número de las mismas. En la figura 8 , se muestra el comportamiento del ruido en corriente, para el sistema agua destilada con partículas de sílice, que muestra cómo el comportamiento de la corriente depende directamente del tamaño de partícula al cual, el material es expuesto bajo condiciones de giro. Para una velocidad de giro de $880 \mathrm{rpm}$, se observaron oscilaciones de mayor amplitud en corriente usando partículas de sílice mayores de $590 \mu \mathrm{m}$, en tanto que para los tamaños menores de 250 y $590 \mu \mathrm{m}$, respectivamente, se obtuvo un comportamiento similar entre ellos pero con menor proporción de cambio en corriente, con respecto a las partículas de mayor tamaño. Para la velocidad de rotación de $1.760 \mathrm{rpm}$, el ruido en corriente en presencia de partículas menores de $250 \mu \mathrm{m}$ es más intenso al inicio del experimento y disminuye su amplitud, alcanzando un valor constante, lo que se relaciona con los primeros impactos sobre la superficie cubierta de óxidos y su destrucción localizada, al inicio de la prueba.

Caso contrario se observa con las partículas de $590 \mu \mathrm{m}$ : al inicio, la amplitud de las oscilaciones fue menor aunque aumentó a medida que la prueba transcurrió. Este comportamiento puede relacionarse con el tamaño mayor de partículas que, al inicio de la prueba, sufren de dificultades para ser arrastradas por el flujo de la rotación y la cantidad de impactos es menor. Sin embargo, una vez que aumenta el volumen de partículas que ingresa al flujo se produce una mayor cantidad de impactos en la superficie metálica. Para el caso de las partículas mayores a $590 \mu \mathrm{m}$, se observan oscilaciones con amplitudes similares al caso anterior, aunque presentan valores transitorios de mayor intensidad, sobrepuestos a la señal base (menor amplitud), lo cual podría explicarse en términos de la presencia de algunas partículas de mayor tamaño que, al incorporarse al flujo e impactarse, producen daños más intensos. 
En la figura 9, se observa el comportamiento del ruido en potencial, complementario a la señal de ruido en corriente, presentado en la figura 8 . El comportamiento del potencial, manifiesta la interacción de partículas sólidas de diferentes tamaños que están rotando bajo dos regímenes de flujo distintoss, laminar y de transición turbulenta, y las superficies de los electrodos. Para la velocidad de giro más baja y para los tamaños de partícula mayores (590 $\mu \mathrm{m}$ y superiores), las oscilaciones de potencial fueron semejantes en amplitud y frecuencia durante toda la prueba. Sin embargo, la amplitud de las oscilaciones para las partículas de menor tamaño $(250 \mu \mathrm{m})$ fue menor. Para la velocidad de giro mayor (1.760 rpm) y para los tamaños de partícula mayores, las oscilaciones de potencial fueron similares en amplitud y frecuencia. Para las partículas de menor tamaño la amplitud fue menor, excepto al final de la prueba donde las oscilaciones aumentaron su amplitud. El comportamiento observado del ruido en potencial podría deberse al efecto de la difusión de especies (oxidrilos) y efectos mecánicos, que se manifiesta mayormente en este parámetro ${ }^{[4,7 \text { y } 16]}$.

Como ejemplo, en la figura 10 , se presenta la resistencia de ruido en función del tiempo, obtenida a partir de la división puntual del ruido en potencial sobre el ruido en corriente, para una velocidad de rotación de $880 \mathrm{rpm}$. Se puede observar, que las partículas de mayor tamaño generaron un valor más constante y pequeño de resistencia de ruido, asociado a un daño mas generalizado de la superficie debido a su tamaño. La resistencia en función del tiempo presenta oscilaciones continuas, a lo largo de la prueba hasta los $2.300 \mathrm{~s}$, disminuyendo la intensidad al final con una resistencia media de dos órdenes de magnitud menor de alrededor de $1 \mathrm{k} \varnothing . \mathrm{cm}^{2}$, respecto a las de menor tamaño. Para estas partículas menores, se presentan valores transitorios de resistencia al inicio y al final de la serie de tiempo, asociados a un ataque más eventual y localizado de la superficie, siendo el comportamiento muy similar (nótese la reproducibilidad) presentando dos picos al inicio y al final de la prueba asociados a efectos localizados. El valor medio de la resistencia está entre 10 y 100 $\mathrm{k} \varnothing . \mathrm{cm}^{2}$. Los valores negativos de la resistencia de ruido se refieren sólo al cambio en la dirección de la corriente en los anillos, debido a que el papel de ánodo y cátodo se invierte ${ }^{[13]}$.

El espectro de resistencia de ruido (impedancia de ruido a baja frecuencia) refleja lo que se obtiene en las series de tiempo de la resistencia de ruido para los diferentes tamaños de partícula, a velocidad de rotación constante (Fig. 10). Se observa que la impedancia de ruido presenta el menor nivel para las mayores partículas y aumenta su valor para las otras dos condiciones ${ }^{[19]}$.
La figura 11 presenta, como ejemplo, los espectros de ruido en corriente y potencial para los diferentes tamaños de partícula a velocidad de rotación constante de $880 \mathrm{rpm}$. Los espectros en corriente se presentan alineados en amplitud en función del tamaño, lo que muestra la relación que hay en la intensidad del ruido en función del tamaño de partícula: a mayor tamaño de partícula mayor es el nivel de ruido ${ }^{[20]}$. Esto, no sucede para el caso del ruido en potencial y no se presenta ningún patrón de comportamiento, aunque por encima de $0,01 \mathrm{mHz}$ la amplitud se hace constante.

El espectro de impedancia obtenido a partir de la división del espectro de voltaje entre el espectro corriente, para diferentes tamaños de partículas de sílice a baja velocidad de rotación ( $880 \mathrm{rpm}$ ) para flujo laminar, se presenta en la figura 12 . Se puede observar que los valores de resistencia de ruido obtenidos, son similares a los que se presenta en la serie de tiempo de la resistencia de ruido (Fig. 10), lo que corrobora el impacto de las partículas sobre la muestra metálica y el daño que provocan las de mayor tamaño ${ }^{[21]}$.

En la figura 13 se presenta el comportamiento de la resistencia de ruido en función del tamaño de partícula y diferente velocidad de rotación. Se puede observar que para la velocidad de rotación baja ( 880 $\mathrm{rpm})$, la resistencia parece casi independiente del tamaño de partícula. Para la velocidad de rotación alta $(1.760 \mathrm{rpm})$ se observa un comportamiento decreciente casi lineal en función del tamaño de partícula $^{[16 \text { y 19] }}$. La resistencia de ruido disminuye a medida que el tamaño de partícula aumenta. Para las partículas de mayor tamaño, la velocidad de rotación alta (régimen de flujo turbulento), permite mover las partículas del fondo de la celda incrementándose

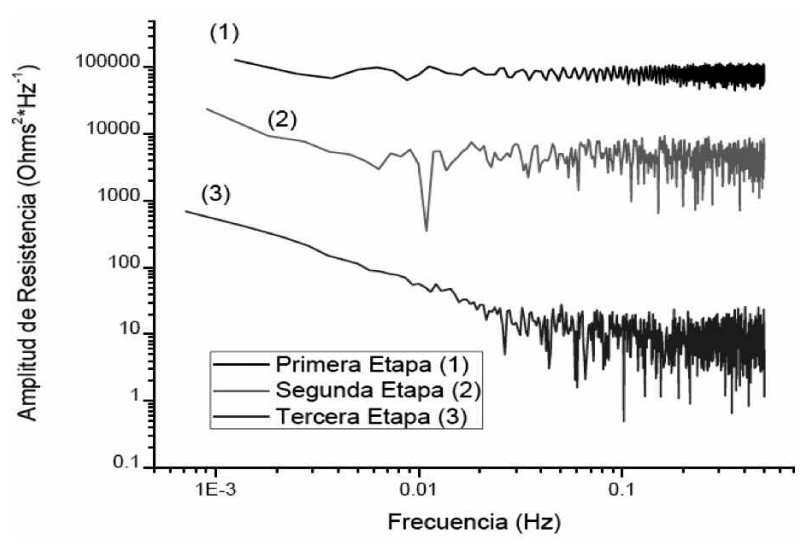

Figura 10. Resistencia de ruido en función del tiempo para diferentes tamaños de partícula de sílice para una velocidad de rotación de 880 rpm.

Figure 10. Noise resistance as a function of time for different particle size and $880 \mathrm{rpm}$ rotation. 
RUIDO ELECTROQUÍMICO DE LA EROSIÓN-CORROSIÓN EN COBRE: SU RELACIÓN CON LOS PARÁMETROS HIDRODINÁMICOS ELECTROCHEMICAL NOISE OF THE EROSION-CORROSION OF COPPER IN RELATION WITH ITS HYDRODYNAMIC PARAMETERS

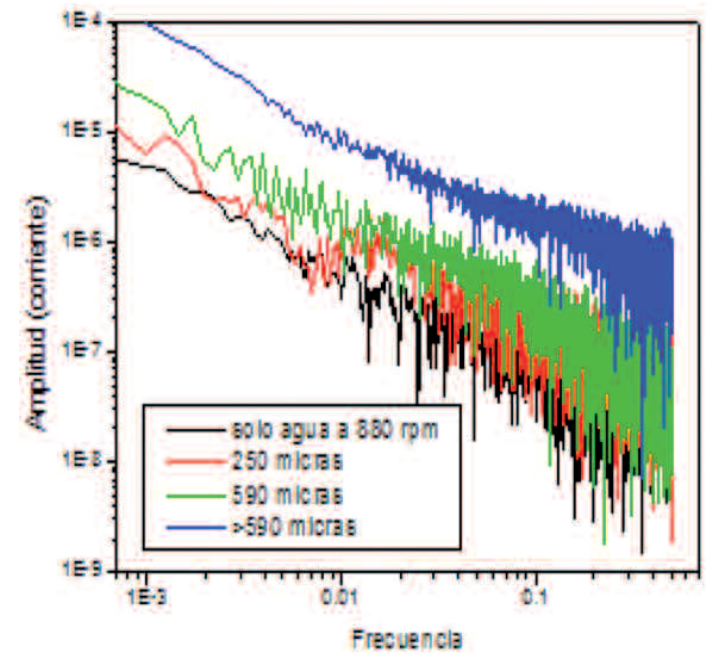

a) Corriente

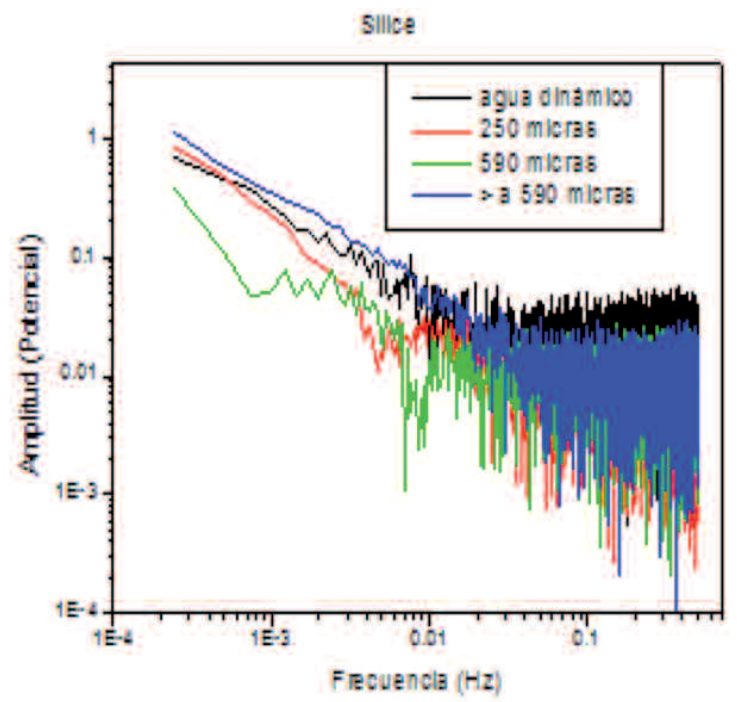

b) Potencial

Figura 11. Espectros de impedancia de ruido en: a) corriente y b) potencial para diferentes tamaños de partícula de sílice para una velocidad de rotación de $880 \mathrm{rpm}$.

Figure 11. Noise impedance spectra for: a) current and b) potential for different silica particle size for a rotation speed of $880 \mathrm{rpm}$.

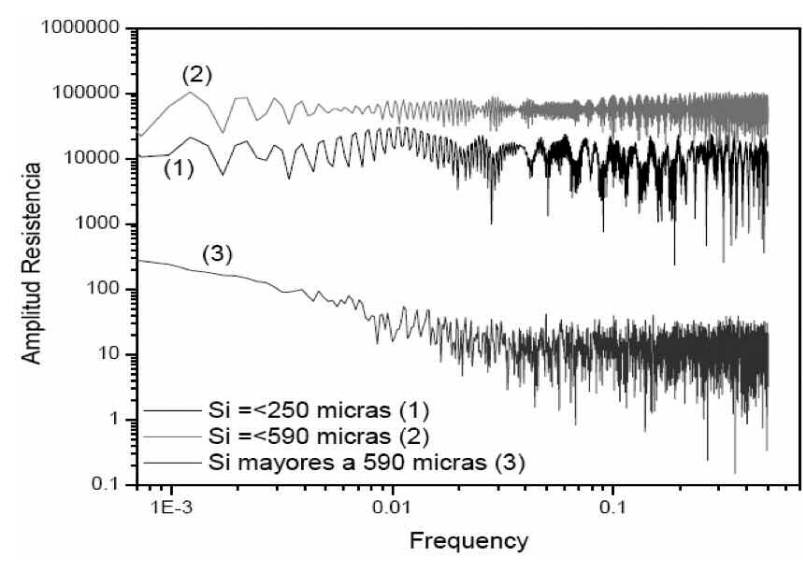

Figura 12. Espectro de impedancia de ruido para diferentes tamaños de partícula de sílice para una velocidad de rotación de $880 \mathrm{rpm}$.

Figure 12. Noise impedance spectra for different silica particle size for a rotation speed of 880 rpm.

los impactos y, consecuentemente, el daño sobre la superficie de los anillos, dificultándose el crecimiento

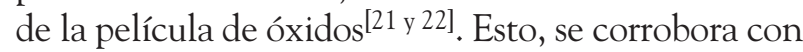
las micrografías de la figura 14, donde se presentan ejemplos de los daños observados sobre la superficie metálica para velocidad alta $(1.760 \mathrm{rpm})$ tamaños y tipos de partícula, presentando erosión e impacta-

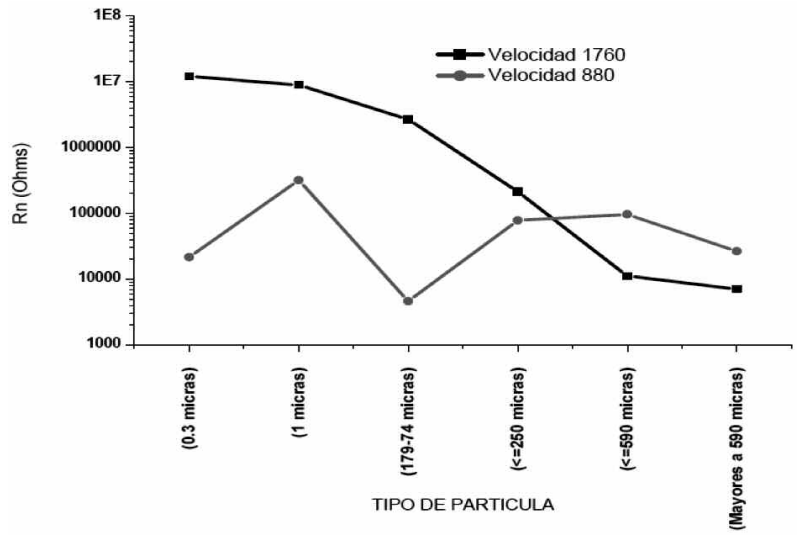

Figura 13. Resistencia de ruido en función del tamaño de partícula y diferente velocidad de rotación.

Figure 13. Noise resistance as a function of particle size and different rotation speed.

ción, que ilustra los resultados obtenidos por medio de la técnica de ruido electroquímico.

\section{CONCLUSIONES}

El fenómeno erosión-corrosión, pudo ser identificado y caracterizado a partir de la técnica electroquímica 


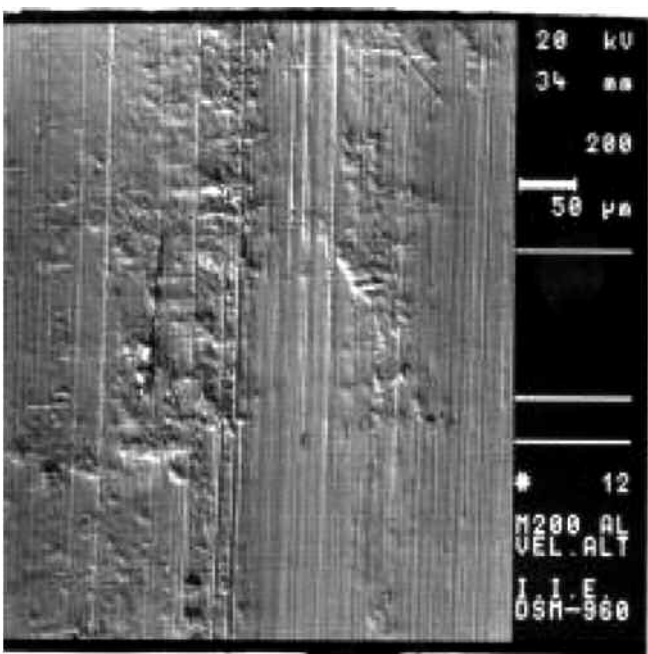

a) Alúmina velocidad alta

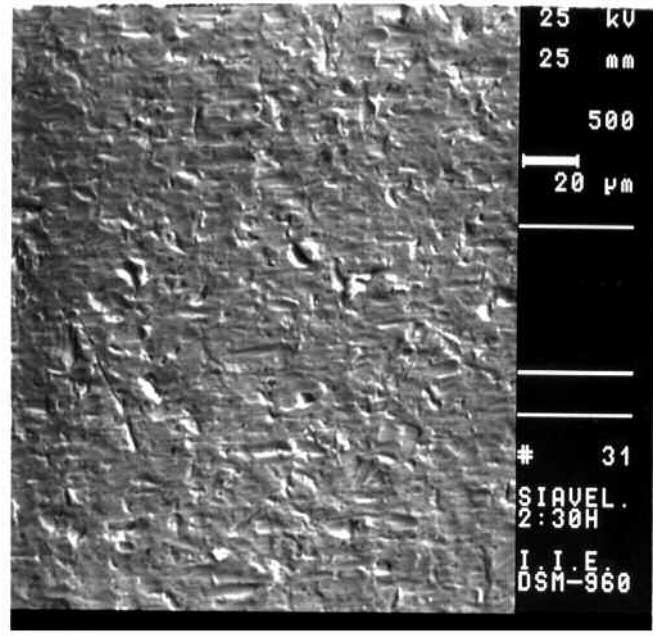

b) Sílice velocidad alta

Figura 14. Daños observados sobre la superficie metálica para velocidad alta (1.760 rpm) y tipo de partícula.

Figure 14. Damage observed over the metal surface for high speed $(1,760 \mathrm{rpm})$ and type of particle.

de ruido, de forma cualitativa y cuantitativa. Con base en los resultados se obtuvieron las siguientes conclusiones:

- Se logró identificar el comportamiento del ruido electroquímico, bajo diferente régimen de flujo, laminar y de transición turbulenta.

- Se identificaron cambios en la señal en función del tamaño de partícula.

- Para la velocidad de rotación baja (flujo laminar) el ruido electroquímico no presenta una relación directa con el tamaño de partícula.

- Para la velocidad de rotación alta (transición turbulenta) el ruido electroquímico presenta una relación directa con el tamaño de partícula.

- La morfología de ataque se relaciona con las condiciones experimentales y se identifica plenamente con el análisis de las señales de ruido electroquímico.

\section{REFERENCIAS}

[1] J. Robbins, Ions in solution: an introduction to electrochemistry, 3rd Edition, Oxford Press, Inglaterra, 1979, pp. 67-78.

[2] J. Allen, L. Bard y R. Faulkner, Electrochemical Method: Fundamentals and Applications, 2nd. Edition, Ed. John Wiley, New York, EE.UU., 1980, pp. 273-278.

[3] J. Uruchurtu y J. L. Dawson, Mater. Sci. Forum (1985) 113-124.
[4] E. Almeida, L. Mariaca, A. Rodriguez y J.Uruchurtu, Electrochemical Noise Measurements for Corrosion Applications STP 1277, Ed. ASTM, New Jersey, EE.UU. 1996, pp. 412-424.

[5] J. M. Malo, J. Uruchurtu y O. M. Corona, Corrosion 58 (2002) 932-940.

[6] G. J. Escalera-Santos, J. Uruchurtu y P. Parmananda, Phys. Rev. Lett. 90 (2003) 1-4.

[7] J. Uruchurtu-Chavarín y J.M. Malo, Trends Corros. Res. 2 (1997) 49-58.

[8] E. Sarmiento, J.G. González-Rodriguez, J.Uruchurtu, O. Sarmiento y C. Menchaca, Int. J. Electrochem. Sci. 4 (2009) 144-155.

[9] L. Mariaca, U. Cano, E. Garcia, J. M. Malo, A. Martinez-Villafañe y J. Uruchurtu, Rev. Metal. Madrid 29 (1993) 284-291.

[10] R. Urzua, J.Siqueiros, L. Morales, I. Rosales y J. Uruchurtu, P. Electrochim. Acta 27 (2009) 127-142.

[11] J.M. Malo y J. Velazco, Electrochemical Noise Measurement, for Corrosion Applications STP 1277, Ed. ASTM, New Jersey, EE. UU., 1996, pp. 387-397.

[12] Y. Puget, K. Threthewey y R. J. Wood, Electrochemical noise analysis of polyurethane-coated steel subjected to erosion-corrosion, Department of Mechanical Engineering, University of Southampton, Highfield, Southampton SO17 1BJ, Inglaterra, 1999.

[13] J.R. Kearns y J.R. Scully, Electrochemical Noise Measurement, for Corrosion Applications STP 
RUIDO ELECTROQUÍMICO DE LA EROSIÓN-CORROSIÓN EN COBRE: SU RELACIÓN CON LOS PARÁMETROS HIDRODINÁMICOS ELECTROCHEMICAL NOISE OF THE EROSION-CORROSION OF COPPER IN RELATION WITH ITS HYDRODYNAMIC PARAMETERS

1277, Ed. ASTM, New Jersey, EE. UU., 1996, pp. 446-471.

[14] R.J.K. Wood, J.A. Wharton, A.J. Speyera and K.S. Tana, School of Engineering Sciences, University of Southampton, Southampton SO17 1BJ, Inglaterra, 2001.

[15] R. Cottis y S. Turgoose, Electrochemical Impedance and Noise, Ed. NACE Internacional, Houston, EE. UU., 1999, pp. 71-93.

[16] J. Botana y M. Marcos, Ruido Electroquímico, Métodos de Análisis, $1^{\underline{a}}$ Edición, Ed. SEPTEM, Oviedo, España, pp. 51-79.

[17] G. Levich, Physicochemical hydrodynamics, $1^{\text {ST }}$ Edition, Prentice Hall, New Jersey, EE.UU., pp. 161-168

[18] P. Roberge y B. Syrett, Erosion-Corrosion, Ed. NACE International, EE. UU., 2004, pp. 34-47.

[19] D.C. Silverman, Corrosion Houston 60 (2004) 1.003-1.023.

[20]. T.Y. Chen, A. A. Moccari y D. D. Macdonald, Corrosion (Houston) 48 (1992) 239-248.
[21] R. W. Staehle, Proc. of Environment-Induced Cracking of Metals, vol. 1, Ed. NACE, Houston, EE. UU., 1989, pp. 561-61.

[22] D. Lopez, J.P. Congote, J.R. Canob, A. Torob, y A.P. Tschiptschin, Wear London 259 (2005) 118-124.

[23] Study of Mass-Transport Limited Corrosion Using Pine Rotated Cylinder Electrodes, Technical Note 2006-01.

[24] P.M. Perillo y R. Haddad, Jornadas SAM - CONAMET - AAS, 2001.

[25] H. S. Klapper, J. Goellner y A. Heyn, Ingeniería \& Desarrollo, 21 (2007).

[26] J.M. Sánchez-Amaya, M. Bethencourt, L. Gonzalez-Rovira y F.J. Botana, Rev. Metal. Madrid 45 (2009) 143-156

[27] E. Milotti, 1/f noise: a pedagogical review, Dipartimento di Fisica, Università di Udine and I.N.F.N, Sezione di Trieste Via delle Scienze, 208, I-33100 Udine, Italy. 